PROCEEDINGS OF THE

AMERICAN MATHEMATICAL SOCIETY

Volume 140, Number 9, September 2012, Pages 3275-3281

S 0002-9939(2012)11161-X

Article electronically published on January 26, 2012

\title{
EXISTENCE OF DOUBLING MEASURES VIA GENERALISED NESTED CUBES
}

\author{
ANTTI KÄENMÄKI, TAPIO RAJALA, AND VILLE SUOMALA
}

(Communicated by Tatiana Toro)

\begin{abstract}
Working on doubling metric spaces, we construct generalised dyadic cubes adapting ultrametric structure. If the space is complete, then the existence of such cubes and the mass distribution principle lead into a simple proof for the existence of doubling measures. As an application, we show that for each $\varepsilon>0$ there is a doubling measure having full measure on a set of packing dimension at most $\varepsilon$.
\end{abstract}

\section{INTRODUCTION AND NOTATION}

A measure $\mu$ on a metric space $(X, d)$ is called doubling if there is a constant $1 \leq D<\infty$ such that

$$
0<\mu(B(x, 2 r)) \leq D \mu(B(x, r))<\infty
$$

for all $x \in X$ and $r>0$. Here $B(x, r)=\{y \in X: d(x, y) \leq r\}$ is a closed ball with centre $x$ and radius $r$. We denote open balls by $U(x, r)$. By a measure we always mean a Borel regular outer measure. A metric space $X$ has the finite doubling property if any ball $B(x, 2 r) \subset X$ may be covered by finitely many balls of radius $r$. Furthermore, such a space is doubling if the number of the $r$-balls needed to cover $B(x, 2 r)$ has an upper bound $N \in \mathbb{N}$ independent of $x$ and $r$.

Let $\mathcal{D}(X)$ be the collection of all doubling measures on $X$. It is clear that if $\mathcal{D}(X) \neq \emptyset$, then $X$ is doubling. The reverse implication is true if $X$ is assumed to be complete. For compact doubling metric spaces this result was first proved by $\mathrm{Vol}^{\prime}$ berg and Konyagin [17, 18]. Luukkainen and Saksman [12] generalised it to the complete case. A slightly simpler proof in the compact case can be found in $\mathrm{Wu}$ [19] (see also Heinonen [6]). Saksman [15] has constructed examples of domains $\Omega$ with $\mathcal{D}(\Omega)=\emptyset$, and the results of Csörnyei and Suomala 2 may be used to study whether $\mathcal{D}(X) \neq \emptyset$ for certain countable sets $X \subset \mathbb{R}$.

In Theorem 2.1 we construct nested families of "cubes" sharing most of the good properties of dyadic (or $r$-adic) cubes of Euclidean spaces. The existence of similar kinds of nested partitions of metric spaces has been studied in many works (see e.g. [11, 14, 1, 8, ). In addition to the fact that our construction adapts ultrametric structure, we believe, given the length of the construction, that it also fulfills the desire of the existence of a simple and effective construction of such cubes (see [14, $\S 7.2])$.

Received by the editors November 19, 2010 and, in revised form, March 30, 2011.

2010 Mathematics Subject Classification. Primary 28C15; Secondary 54E50.

Key words and phrases. Doubling measure, nested cubes in metric spaces.

The third author acknowledges the support of the Academy of Finland, project \#126976.

(C)2012 American Mathematical Society 
As an application, we show in Theorem 3.1 that complete doubling metric spaces carry doubling measures. This is of course a known result (see [18, 19, 12]). Since our proof uses nested partitions and the mass distribution principle, we are able to get the result directly also in the unbounded case; the extra step in the unbounded case (see [12]) is not needed. Also, once the partitions have been fixed, our method can be used to construct doubling measures possessing certain measure theoretical self-similarity; see Remark 5.1(3). In fact, in Theorem 4.1 we show that for each $\varepsilon>0$ there is a doubling measure having full measure on a set of packing dimension at most $\varepsilon$. This result was known before only for the Hausdorff dimension (see [16, 19]).

In what follows, the (metric) closure, interior and boundary of a set $A \subset X$ are denoted by $\bar{A}, \operatorname{int}(A)$ and $\partial A$, respectively.

\section{Construction of generalised Dyadic cubes}

Theorem 2.1. If $X$ is a metric space with the finite doubling property and $0<r<$ $\frac{1}{3}$, then there exists a collection $\left\{Q_{k, i}: k \in \mathbb{Z}, i \in N_{k} \subset \mathbb{N}\right\}$ of Borel sets having the following properties:

(1) $X=\bigcup_{i \in N_{k}} Q_{k, i}$ for every $k \in \mathbb{Z}$,

(2) $Q_{k, i} \cap Q_{m, j}=\emptyset$ or $Q_{k, i} \subset Q_{m, j}$ when $k, m \in \mathbb{Z}, k \geq m, i \in N_{k}$ and $j \in N_{m}$,

(3) for every $k \in \mathbb{Z}$ and $i \in N_{k}$ there exists a point $x_{k, i} \in X$ so that

$$
U\left(x_{k, i}, c r^{k}\right) \subset Q_{k, i} \subset B\left(x_{k, i}, C r^{k}\right)
$$

where $c=\frac{1}{2}-\frac{r}{1-r}$ and $C=\frac{1}{1-r}$,

(4) there exists a point $x_{0} \in X$ so that for every $k \in \mathbb{Z}$ there is $i \in N_{k}$ so that

$$
U\left(x_{0}, c r^{k}\right) \subset Q_{k, i},
$$

(5) $\left\{x_{k, i}: i \in N_{k}\right\} \subset\left\{x_{k+1, i}: i \in N_{k+1}\right\}$ for all $k \in \mathbb{Z}$.

Proof. Fix a point $x_{0} \in X$ and start by choosing a maximal collection of points $\left\{x_{0, i}: i \in N_{0}\right\} \subset X$ containing $x_{0}$ and having the property that $d\left(x_{0, i}, x_{0, j}\right) \geq 1$ if $i \neq j$. Next, for each $k \in \mathbb{N}$, let $\left\{x_{k, i}: i \in N_{k}\right\} \supset\left\{x_{k-1, i}: i \in N_{k-1}\right\}$ be a maximal collection of points having mutual distances at least $r^{k}$. If $k \in \mathbb{Z}, k<0$, we let $\left\{x_{k, i}: i \in N_{k}\right\}$ be any maximal subcollection of $\left\{x_{k+1, i}: i \in N_{k+1}\right\}$ containing $x_{0}$ whose points have mutual distances at least $r^{k-1}$.

In the set of all possible pairs $(k, i), k \in \mathbb{Z}, i \in N_{k}$, consider the smallest partial order $\prec$ that satisfies the following property: For each $k \in \mathbb{Z}$ and $i \in N_{k+1}$, we have $(k+1, i) \prec(k, j)$ if

$$
j=\min \left\{h \in N_{k}: \operatorname{dist}\left(x_{k+1, i}, x_{k, h}\right)=\min _{l \in N_{k}} \operatorname{dist}\left(x_{k+1, i}, x_{k, l}\right)\right\} .
$$

Notice that $\min _{l \in N_{k}} \operatorname{dist}\left(x_{k+1, i}, x_{k, l}\right)$ exists, because $X$ has the finite doubling property, and that $j$ also exists and is unique. The sets $Q_{k, i}$ will be defined by using this partial order.

We first define the sets $Q_{0, i}$ for $i \in N_{0}$ as

$$
Q_{0, i}=\overline{\left\{x_{l, j}:(l, j) \prec(0, i)\right\}} \backslash \bigcup_{j<i} Q_{0, j} .
$$

For $k<0$ we define the sets $Q_{k, i}$ inductively as

$$
Q_{k, i}=\bigcup_{(k+1, j) \prec(k, i)} Q_{k+1, j},
$$


whereas for $k>0$, we let

$$
Q_{k, i}=Q_{k-1, j} \cap \overline{\left\{x_{l, j}:(l, j) \prec(k, i)\right\}} \backslash \bigcup_{j<i} Q_{k, j},
$$

where $(k, i) \prec(k-1, j)$. The defined sets are clearly Borel.

Let us check that the sets $Q_{k, i}$ satisfy the conditions (11)-(5). To see (11) it is enough to notice that

$$
\bigcup_{i \in N_{k}} Q_{k, i}=\bigcup_{i \in N_{k}} \overline{\left\{x_{l, j}:(l, j) \prec(k, i)\right\}},
$$

which is dense and closed in $X$. Conditions (2) and (5) follow immediately from the construction. Let us next verify (3). The fact that $\left\{x_{m, i}: i \in N_{m}\right\}$ is a maximal $r^{m}$-separated subset of $\left\{x_{m+1, j}: j \in N_{m+1}\right\}$ together with the definition of $\prec$ implies that $d\left(x_{m, i}, x_{m+1, j}\right) \leq r^{m}$ if $(m+1, j) \prec(m, i)$. This gives

$$
Q_{k, i} \subset B\left(x_{k, i}, \sum_{m=k}^{\infty} r^{m}\right)=B\left(x_{k, i}, \frac{1}{1-r} r^{k}\right) .
$$

On the other hand, if $n>k$ and $(n, j) \nprec(k, i)$, then $d\left(x_{k, i}, x_{n, j}\right) \geq \frac{1}{2} r^{k}-$ $\sum_{m=k+1}^{\infty} r^{m}=\left(\frac{1}{2}-\frac{r}{1-r}\right) r^{k}$. This implies

$$
U\left(x_{k, i},\left(\frac{1}{2}-\frac{r}{1-r}\right) r^{k}\right) \subset Q_{k, i}
$$

and finishes the proof of (3). Finally, claim (4) follows from (3) since $x_{0} \in\left\{x_{k, i}\right.$ : $\left.i \in N_{k}\right\}$ for all $k \in \mathbb{Z}$.

Remark 2.2. The statement of Theorem 2.1 remains true also for any $\frac{1}{3} \leq r<1$ with $\frac{1}{2}-\frac{r}{1-r}$ and $\frac{1}{1-r}$ replaced by some constants $0<c<C<\infty$ depending only on $r$. This can be easily seen as follows: First apply Theorem 2.1 with $r=\frac{1}{4}$ to obtain the families $\left\{Q_{k, i}: i \in N_{k}\right\}$. Then, for any $\frac{1}{3} \leq \tilde{r}<1$ and $n \in \mathbb{N}$, we choose $k=k(n, \tilde{r}) \in \mathbb{N}$ such that $4^{-k}<\tilde{r}^{n} \leq 4^{-k+1}$. Now $\left\{\widetilde{Q}_{n, i}\right\}=\left\{Q_{k(n, \tilde{r}), i}:\right.$ $\left.i \in N_{k(n, \tilde{r})}\right\}$ are the desired families. We formulated the result as in Theorem 2.1 since the explicit expressions for the constants $c$ and $C$ for small $r$ are needed in the proof of Theorem 3.1 below.

\section{EXISTENCE OF DOUBLING MEASURES}

Theorem 3.1. If $X \neq \emptyset$ is a complete doubling metric space, then $\mathcal{D}(X) \neq \emptyset$.

Proof. Fix $0<r \leq \frac{1}{7}$ and let $\mathcal{Q}=\left\{Q_{k, i}: k \in \mathbb{Z}, i \in N_{k}\right\},\left\{x_{k, i}: k \in \mathbb{Z}, i \in N_{k}\right\}$, and the constants $0<c<C<\infty$ be as in Theorem 2.1. Let

$$
M_{k, i}=\#\left\{j \in N_{k+1}: Q_{k+1, j} \subset Q_{k, i}\right\}-1 .
$$

Since $X$ is doubling, it follows using Theorem 2.12(2) and (3) that there exists $M \in \mathbb{N}$ such that $M_{k, i} \leq M$ for every $k \in \mathbb{Z}$ and $i \in N_{k}$. We define a code tree $\Sigma$ by setting $\Sigma=\left\{\left(i_{k}\right)_{k \in \mathbb{Z}}: Q_{k, i_{k}} \subset Q_{k-1, i_{k-1}}\right.$ for all $\left.k \in \mathbb{Z}\right\}$ and equip this with the usual ultrametric: the distance between two different codes $\left(i_{k}\right)$ and $\left(j_{k}\right)$ is $2^{-n}$, where $n$ is the first index at which the codes differ, $n=\min \left\{k: i_{k} \neq j_{k}\right\}$. Theorem 2.1(2) and (4) guarantee that this metric is well defined. We also define cylinders $[k, i]=\left\{\left(j_{n}\right)_{n \in \mathbb{Z}} \in \Sigma: j_{k}=i\right\}$ and set $\Sigma_{*}=\{[k, i]: k \in \mathbb{Z}$ and $i \in$ $\left.N_{k}\right\}$. Since $X$ is complete, we may define a projection $\pi: \Sigma \rightarrow X$ by the relation 
$\left\{\pi\left(\left(i_{k}\right)_{k \in \mathbb{Z}}\right)\right\}=\bigcap_{k \in \mathbb{Z}} \overline{Q_{k, i_{k}}}$. Now we clearly have $\pi([k, i])=\overline{Q_{k, i}}$ for every $k \in \mathbb{Z}$ and $i \in N_{k}$.

We define a set function $\nu: \Sigma_{*} \rightarrow[0, \infty)$ by first choosing $0<p<1 /(M+1)$, $i_{0} \in N_{0}$ and setting $\nu\left(\left[0, i_{0}\right]\right)=1$, and then requiring that for every $k \in \mathbb{Z}$ and $i \in N_{k}$ we have

$$
\nu([k+1, i])= \begin{cases}p \nu([k, j]), & \text { if } Q_{k+1, i} \subset Q_{k, j} \text { and } x_{k+1, i} \neq x_{k, j}, \\ \left(1-M_{k, i} p\right) \nu([k, j]), & \text { if } Q_{k+1, i} \subset Q_{k, j} \text { and } x_{k+1, i}=x_{k, j} .\end{cases}
$$

We may now easily extend $\nu$ to a measure on $\Sigma$ by setting

$$
\nu(A)=\inf \left\{\sum_{j} \nu\left(\left[k_{j}, i_{j}\right]\right): A \subset \bigcup_{j}\left[k_{j}, i_{j}\right]\right\}
$$

for all $A \subset X$. The main reason for this to work is the fact that the cylinders $[k, j]$ are both open and closed (compact) in $\Sigma$. See also [5, §10]. It follows immediately from the construction that $\nu$ is a doubling measure on $\Sigma$.

Let $\mu=\pi \nu$ be the projected measure on $X$ given by $\mu(A)=\nu\left(\pi^{-1}(A)\right)$ for all $A \subset X$. It is then clear that we have the estimates

$$
\mu\left(\operatorname{int}\left(Q_{k, i}\right)\right) \leq \nu([k, i]) \leq \mu\left(\overline{Q_{k, i}}\right)
$$

for every $k \in \mathbb{Z}$ and $i \in N_{k}$. Let us next show that this can be sharpened to

$$
\mu\left(Q_{k, i}\right)=\nu([k, i]) .
$$

Fix $k \in \mathbb{Z}, i \in N_{k}$ and $\left(j_{n}\right)_{n \in \mathbb{Z}} \in \pi^{-1}\left(\partial Q_{k, i}\right)$. Then $x:=\pi\left(\left(j_{n}\right)_{n \in \mathbb{Z}}\right) \in \overline{Q_{n, j_{n}}}$ for every $n \in \mathbb{Z}$. Theorem 2.1(3), together with the fact $r \leq 1 / 7$, implies that

$$
\overline{Q_{n+1, l_{n+1}}} \subset B\left(x_{n, j_{n}}, C r^{n+1}\right) \subset U\left(x_{n, j_{n}}, c r^{n}\right) \subset Q_{n, j_{n}}
$$

for every $n \in \mathbb{Z}$, where $l_{n+1} \in N_{n+1}$ satisfies $x_{n+1, l_{n+1}}=x_{n, j_{n}}$. Hence $Q_{n+1, l_{n+1}} \cap$ $\partial Q_{k, i}=\emptyset$, and so $\left[n+1, l_{n+1}\right] \subset\left[n, j_{n}\right] \backslash \pi^{-1}\left(\partial Q_{k, i}\right)$ for every $n \in \mathbb{Z}$. This means that $\pi^{-1}\left(\partial Q_{k, i}\right)$ is a porous subset of $\Sigma$, and since $\nu$ is doubling, it now follows that $\mu\left(\partial Q_{k, i}\right)=\nu\left(\pi^{-1}\left(\partial Q_{k, i}\right)\right)=0$ (see e.g. [9, Proposition 3.4] for a proof of this elementary fact). Combining this with (3.3) implies (3.4).

It remains to show that $\mu$ is doubling. If $y \in X$ and $t>0$, let $k \in \mathbb{Z}$ be such that $3 r^{k} \leq t<3 r^{k-1}$. By Theorem 2.1(3), the ball $B(y, t)$ contains $Q_{k, i}$ for some $i \in N_{k}$. On the other hand, the ball $B(y, 2 t)$ intersects $Q_{k, j}$ for at most $\tilde{M}$ indices $j \in N_{k}$, where $\tilde{M}<\infty$ depends only on $r$ and the doubling constant $N$ of $X$. Thus it suffices to show that there exists a constant $1 \leq \tilde{C}<\infty$ so that

$$
\mu\left(Q_{k, j}\right) \leq \tilde{C} \mu\left(Q_{k, i}\right)
$$

whenever $Q_{k, j} \cap B(y, 2 t) \neq \emptyset$. Fix $j \in N_{k}$ for which $Q_{k, j} \cap B(y, 2 t) \neq \emptyset$. We may assume that $i \neq j$ as otherwise (3.5) holds trivially. Observe that

$$
d\left(x_{k, i}, x_{k, j}\right) \leq 3 t+C r^{k}<r^{k-3} .
$$

Let $m$ be the largest integer such that $Q_{k, i} \cup Q_{k, j} \subset Q_{m, l}$ for some $l \in N_{m}$. For each $m \leq n \leq k$ let $i_{n}, j_{n} \in N_{n}$ be the indices that satisfy $Q_{k, i} \subset Q_{n, i_{n}}$ and $Q_{k, j} \subset Q_{n, j_{n}}$. If $m<n \leq k-4$, it follows that

$$
x_{n, j_{n}} \neq x_{n+1, j_{n+1}} \text { and } x_{n, i_{n}} \neq x_{n+1, i_{n+1}}
$$

as otherwise Theorem 2.1 implies (recall $r \leq \frac{1}{7}$ )

$$
d\left(x_{k, i}, x_{k, j}\right)>c r^{n}-C r^{n+1}=\left(\frac{1}{2}-\frac{2 r}{1-r}\right) r^{n} \geq r^{n+1} \geq r^{k-3},
$$


contrary to (3.6). Now (3.4), (3.7), and (3.2) imply that $\mu\left(Q_{n, j_{n}}\right)=p \mu\left(Q_{n+1, j_{n+1}}\right)$ and $\mu\left(Q_{n, i_{n}}\right)=p \mu\left(Q_{n+1, i_{n+1}}\right)$ and thus

$$
\frac{\mu\left(Q_{n+1, j_{n+1}}\right)}{\mu\left(Q_{n, j_{n}}\right)} \frac{\mu\left(Q_{n, i_{n}}\right)}{\mu\left(Q_{n+1, i_{n+1}}\right)}=1
$$

for $m<n \leq k-4$. Hence

$$
\frac{\mu\left(Q_{k, j}\right)}{\mu\left(Q_{k, i}\right)}=\prod_{n=m}^{k-1} \frac{\mu\left(Q_{n+1, j_{n+1}}\right)}{\mu\left(Q_{n, j_{n}}\right)} \frac{\mu\left(Q_{n, i_{n}}\right)}{\mu\left(Q_{n+1, i_{n+1}}\right)} \leq p^{-4},
$$

giving (3.5) and finishing the proof.

\section{Dimension of Doubling measures}

The local $L^{q}$-spectrum was recently introduced in [10]. It gives one way to quantify the local homogeneity of a given measure and can also be used to estimate the local dimensions.

Suppose that $\mu$ is a measure on a doubling metric space $X$ so that bounded sets have finite measure. Denote the support of $\mu$ by $\operatorname{spt}(\mu)$. If $0<r<1, x \in \operatorname{spt}(\mu)$, and $q \geq 0$, then, following [10, we define the local $L^{q}$-spectrum of $\mu$ at $x$ by setting

$$
\tau_{q}(\mu, x)=\lim _{t \downarrow 0} \liminf _{k \rightarrow \infty} \frac{\log \sum_{Q \in \mathcal{Q}_{k}(x, t)} \mu(Q)^{q}}{k \log r},
$$

where $\mathcal{Q}_{k}(x, t)=\left\{Q_{k, i} \cap B(x, t): i \in N_{k}\right\}$ and the collection $\left\{Q_{k, i}: k \in \mathbb{Z}, i \in N_{k}\right\}$ is as in Theorem 2.1

Let

$$
\overline{\operatorname{dim}}_{\mathrm{loc}}(\mu, x)=\limsup _{t \downarrow 0} \frac{\log \mu(B(x, t))}{\log t}
$$

be the upper local dimension of $\mu$ at $x$. If instead of $\lim \sup _{t \downarrow 0}$ we take $\lim \inf _{t \downarrow 0}$, we get the lower local dimension of $\mu$ at $x$, denoted by $\underline{\operatorname{dim}}_{\mathrm{loc}}(\mu, x)$ (see [3, §10.1]). According to [10], we have

$$
\lim _{q \downarrow 1} \frac{\tau_{q}(\mu, x)}{q-1} \leq \underline{\operatorname{dim}}_{\mathrm{loc}}(\mu, x) \leq \overline{\operatorname{dim}}_{\mathrm{loc}}(\mu, x) \leq \lim _{q \uparrow 1} \frac{\tau_{q}(\mu, x)}{q-1}
$$

for $\mu$-almost all $x \in X$. This estimate generalises the results [13, Theorem 1.1], [7, Theorems 1.3 and 4.1], and [4, Theorem 1.4].

We now apply (4.1) for the measures constructed in the proof of Theorem 3.1 to estimate their packing dimension.

Theorem 4.1. If $X \neq \emptyset$ is a complete doubling metric space, then for every $\varepsilon>0$ there is a doubling measure $\mu$ on $X$ such that

$$
\overline{\operatorname{dim}}_{\mathrm{loc}}(\mu, x) \leq \varepsilon
$$

for $\mu$-almost all $x \in X$.

Proof. Fix $0<r \leq \frac{1}{7}$ and let $\mathcal{Q}=\left\{Q_{k, i}: k \in \mathbb{Z}, i \in N_{k}\right\}$ be as in Theorem 2.1. Let $\mu$ be the measure constructed in the proof of Theorem 3.1. Fix $k \in \mathbb{Z}$ and $i \in N_{k}$ and let $M_{k, i}$ be as in (3.1). Since $M_{k, i} \leq M$ and $p \leq \frac{1}{M_{k, i}+1}$, we get 
$M_{k, i} p^{q}+\left(1-M_{k, i} p\right)^{q} \leq M p^{q}+(1-M p)^{q}$ for all $0<q<1$. Recalling (3.2) and (3.5), this implies

$$
\begin{aligned}
\sum_{Q_{k+1, j} \subset Q_{k, i}} \mu\left(Q_{k+1, j}\right)^{q} & =\mu\left(Q_{k, i}\right)^{q}\left(M_{k, i} p^{q}+\left(1-M_{k, i} p\right)^{q}\right) \\
& \leq \mu\left(Q_{k, i}\right)\left(M p^{q}+(1-M p)^{q}\right) .
\end{aligned}
$$

Using this estimate recursively leads to $\tau_{q}(\mu, x) \geq \log \left(M p^{q}+(1-M p)^{q}\right) / \log r$ for all $x \in X$ and $0<q<1$. Combining this with (4.1) gives

$$
\overline{\operatorname{dim}}_{\mathrm{loc}}(\mu, x) \leq \lim _{q \uparrow 1} \frac{\tau_{q}(\mu, x)}{q-1} \leq \frac{M p \log p+(1-M p) \log (1-M p)}{\log r}
$$

for $\mu$-almost all $x \in X$.

As the upper bound in (4.2) can be made arbitrarily small by choosing $p>0$ small enough in (3.2), we have shown the claim.

\section{FURTHER REMARKS}

Remark 5.1. (1) It is tempting to try to define the measure $\mu$ in the proof of Theorem 3.1 directly without going into the code space $\Sigma$. More precisely, first define $\tilde{\mu}\left(Q_{k, i}\right)$ as $\nu([k, i])$ in (3.2) and then let $\mu(A)=\inf \left\{\sum_{j} \tilde{\mu}\left(Q_{k_{j}, i_{j}}\right): A \subset\right.$ $\left.\bigcup_{j} Q_{k_{j}, i_{j}}\right\}$ for $A \subset X$. Although it now follows from the proof of Theorem 3.1 it is not a priori clear that $\mu\left(Q_{k, i}\right)=\tilde{\mu}\left(Q_{k, i}\right)$ for all $k$ and $i$. Observe that in the code space $\Sigma$ this is not a problem since the cylinders $[k, i]$ are both open and compact.

(2) The authors of the articles [18, 12] prove not only the existence of doubling measures but also the existence of $\alpha$-homogeneous measures for each $\alpha$ strictly larger than the Assouad dimension of $X$ (see e.g. [6] for the definitions). It is an easy exercise to check that given such an $\alpha$, if $r>0$ is small enough and $p=r^{-\beta}$ in the proof of Theorem 3.1 above, where $\beta$ is between $\alpha$ and the Assouad dimension of $X$, then the measure $\mu$ will be $\alpha$-homogeneous.

(3) Using precisely the same idea as in the proof of Theorem 3.1 one can define more general "self-similar" type doubling measures on $X$. Suppose for instance that our space $X$ is such that the number of descendants of each cube $Q_{k, i}$ is at least $n \in \mathbb{N}$. Let $p_{1}, \ldots, p_{n}>0$ be positive numbers with $\sum_{i=1}^{n} p_{i}=1$ and fix $0<p<M^{-2}$. Instead of (3.2), we distribute the measure of $Q_{k, i}$ among the descendants in the following way: For each $1 \leq m \leq n$ choose $j_{m} \in N_{k+1}$ so that $Q_{k+1, j_{m}} \subset Q_{k, i}$ and $j_{m} \neq j_{l}$ when $m \neq l$. Define $\mu\left(Q_{k+2, j}\right)=p \mu\left(Q_{k, i}\right)$ if $Q_{k+2, j} \subset Q_{k, i}$ and $x_{k+2, j} \notin\left\{x_{k+1, j_{1}}, \ldots, x_{k+1, j_{n}}\right\}$. Then divide the rest of the measure of $\mu\left(Q_{k, i}\right)$ among the "central subcubes" of $Q_{k, j_{1}}, \ldots, Q_{k, j_{n}}$ according to the probabilities $p_{1}, \ldots, p_{n}$.

\section{ACKNOWLEDGEMENT}

The authors thank the referee for useful comments.

\section{REFERENCES}

[1] M. Christ. A $T(b)$ theorem with remarks on analytic capacity and the Cauchy integral. Colloq. Math., 60/61(2):601-628, 1990. MR1096400 (92k:42020)

[2] M. Csörnyei and V. Suomala. Cantor sets and doubling measures. Work in progress, 2010.

[3] K. J. Falconer. Techniques in Fractal Geometry. John Wiley \& Sons Ltd., England, 1997. MR.1449135 (99f:28013) 
[4] A.-H. Fan, K.-S. Lau, and H. Rao. Relationships between different dimensions of a measure. Monatsh. Math., 135(3):191-201, 2002. MR.1897575 (2003g:28014)

[5] P. R. Halmos. Measure Theory. D. Van Nostrand Company, Inc., New York, 1950. MR0033869 (11:504d)

[6] J. Heinonen. Lectures on analysis on metric spaces. Universitext, Springer-Verlag, New York, 2001. MR 1800917 (2002c:30028)

[7] Y. Heurteaux. Estimations de la dimension inférieure et de la dimension supérieure des mesures. Ann. Inst. H. Poincaré Probab. Statist., 34(3):309-338, 1998. MR1625871 (99g:28013)

[8] T. Hytönen and H. Martikainen. Non-homogeneous Tb theorem and random dyadic cubes on metric measure spaces. Preprint. arXiv:0911.4387, 2009.

[9] E. Järvenpää, M. Järvenpää, A. Käenmäki, T. Rajala, S. Rogovin, and V. Suomala. Packing dimension and Ahlfors regularity of porous sets in metric spaces. Math. Z., 266(1):83-105, 2010. MR 2670673

[10] A. Käenmäki, T. Rajala, and V. Suomala. Local homogeneity and dimension of measures in doubling metric spaces. Preprint. arXiv:1003.2895, 2010.

[11] D. G. Larman. On Hausdorff measure in finite-dimensional compact metric spaces. Proc. London Math. Soc. (3), 17:193-206, 1967. MR0210874 (35:1759)

[12] J. Luukkainen and E. Saksman. Every complete doubling metric space carries a doubling measure. Proc. Amer. Math. Soc., 126(2):531-534, 1998. MR.1443161 (99c:28009)

[13] S.-M. Ngai. A dimension result arising from the $L^{q}$-spectrum of a measure. Proc. Amer. Math. Soc., 125(10):2943-2951, 1997. MR 1402878 (97m:28007)

[14] C. A. Rogers. Hausdorff measures. Cambridge University Press, London, 1970. MR0281862 $(43: 7576)$

[15] E. Saksman. Remarks on the nonexistence of doubling measures. Ann. Acad. Sci. Fenn. Math., 24(1):155-163, 1999. MR1678044 (2000b:28006)

[16] P. Tukia. Hausdorff dimension and quasisymmetric mappings, Math. Scand., 65(1):152-160, 1989. MR $1051832(92 \mathrm{~b}: 30026)$

[17] A. L. Vol'berg and S. V. Konyagin. A homogeneous measure exists on any compactum in $\mathbf{R}^{n}$. Dokl. Akad. Nauk SSSR, 278(4):783-786, 1984. MR765294 (86d:28018)

[18] A. L. Vol'berg and S. V. Konyagin. On measures with the doubling condition. Izv. Akad. Nauk SSSR Ser. Mat., 51(3):666-675, 1987. MR903629 (88i:28006)

[19] J.-M. Wu. Hausdorff dimension and doubling measures on metric spaces. Proc. Amer. Math. Soc., 126(5):1453-1459, 1998. MR1443418(99h:28016)

Department of Mathematics and Statistics, P.O. Box 35 (MaD), FI-40014 University OF JYVÄSKYlä, Finland

E-mail address: antti.kaenmaki@jyu.fi

Scuola Normale Superiore, Piazza dei Cavalieri 7, I56127 Pisa, Italy

E-mail address: tapio.rajala@sns.it

Department of Mathematics and Statistics, P.O. Box 35 (MaD), FI-40014 University oF JYVÄSKYLÄ, FinLAND

E-mail address: ville.suomala@jyu.fi

Current address: Department of Mathematics and Statistics, P.O. Box 3000, FI-90014 University of Oulu, Finland

E-mail address: ville.suomala@oulu.fi 